\title{
Bemerkungen zur numerischen Berechnung von Molekülintegralen bei kleinem Kernabstand
}

\author{
Von Werner Bingel \\ Aus der Forschungsstelle für Spektroskopie in der Max-Planck-Gesellschaft, Hechingen \\ (Z. Naturforschg. 11 a, 85-88 [1956] ; eingegangen am 19. November 1955)
}

\begin{abstract}
Für die Übergangsintegrale $K_{\alpha \beta}$ wird eine neue Darstellung durch Hilfsfunktionen $C_{n}(\varrho)$ angegeben, die für die numerische Berechnung bei kleinem $\varrho$ zweckmäßiger ist als die bisherigen Darstellungen. Eine neu berechnete Tabelle der $C_{n}$ wird angegeben. Es wird gezeigt, daß sich auch die Interpolation der $K$-Integrale besser mit den $C$-Funktionen durchführen läßt.
\end{abstract}

Die Ergebnisse jeder quantenmechanischen Behandlung eines Moleküls lassen sich durch eine Reihe von Integralen (Coulomb-, Austausch-, Übergangsintegrale usw.) ausdrücken. Diese Integrale sind Funktionen der Kernabstände $R$ im Molekül und der effektiven Kernladungen $Z_{a}$ der verwendeten Einelektronenfunktionen $\psi_{a}$ (AO's). Für diese Integrale existieren in der Literatur Formeln ${ }^{1-4}$, durch die sie auf gewisse Hilfsfunktionen zurückgeführt werden, die tabuliert sind $^{1}$. Zum Teil sind auch die Integrale selbst tabuliert ${ }^{1,3,4}$. Der Variabilitätsbereich von $\varrho$ erstreckt sich bei diesen Tafeln meist von $\varrho=1$ bis etwa 10 mit einer Spanne von 0,25 oder 0,5. Im Verlaufe von Molekülberechnungen, die vom vereinigten Atom ausgehen, erwies es sich als notwendig, speziell das Übergangsintegral (nuclear attraction integral)

$$
K_{\alpha \beta}(R)=\int \frac{a_{a}(1) a_{\beta}(1)}{r_{\mathrm{b} 1}} \mathrm{~d} \tau_{1} \quad \text { (in atomaren Einheiten) }
$$

für kleine Werte von $R$ zu kennen. Gl. (1) stellt anschaulich die potentielle Energie einer Ladungsverteilung $a_{\alpha}(1) \cdot a_{\beta}(1)$ um das Atom a im CoulombFeld einer Punktladung beim Atom $\mathrm{b}$ dar. $R$ ist der Abstand der Atome a und b, $r_{\mathrm{b} 1}$ der Abstand des Integrationspunktes 1 vom Atom b. Für $R \rightarrow 0$ geht (1) in das Einzentren-Übergangsintegral ${ }^{5}$

$$
K_{\alpha \beta}^{0}=K_{\alpha \beta}(0)=\int \frac{a_{\alpha}(1) a_{\beta}(1)}{r_{\mathrm{a} 1}} \mathrm{~d} \tau_{1}
$$

über, für $R \rightarrow \infty$ geht $K \rightarrow 0$. Dieses Integral ist bisher so ausgewertet worden, daß man elliptische Koordinaten $\xi, \eta$ mit den Zentren in a und b ein-

1 M. K o t a n i u. Mitarb., Proc. Phys. Math. Soc. Japan, Third Series, 20 [1938] u. 22 [1940].

${ }^{2}$ C. C. J. R o ot ha a n, J. Chem. Phys. 19, 1445 [1951].

3 H. J. K o p in e c k, Z. Naturforschg. 5 a, 420 [1950]; 7 a, 785 [1952]. führt. $K_{\alpha \beta}$ läßt sich dann durch die folgenden Hilfsfunktionen ausdrücken

$$
\begin{array}{r}
A_{n}(\varrho)=\int_{1}^{\infty} \xi^{n} e^{-\varrho \xi} \mathrm{d} \xi=\frac{n !}{\varrho^{n+1}} e^{-\varrho} \sum_{k=0}^{n} \frac{\varrho^{k}}{k !}, \quad(2 \mathrm{a}) \\
B_{n}(\varrho) \quad(2 \mathrm{~b}) \\
=\int_{-1}^{+1} \eta^{n} e^{-\varrho \eta} \mathrm{d} \eta=-A_{n}(\varrho)-(-1)^{n} A_{n}(-\varrho) .
\end{array}
$$

So ist z. B. $K_{2 \mathrm{~s} 2 \mathrm{~s}}$ bei gleicher effektiver Kernladung $Z_{2 \mathrm{~s}}$ der beiden Funktionen $\left(\zeta_{2 \mathrm{~s}}=Z_{2 \mathrm{~s}} / 2, \varrho=\zeta_{2 \mathrm{~s}} R\right)$ für Slater-AO's

$$
\frac{K_{2 \mathrm{~s} 2 \mathrm{~s}}(\varrho)}{\zeta_{2 \mathrm{~s}}}=\frac{4}{3} \varrho^{4}\left\{\frac{4 !}{(2 \varrho)^{5}}+A_{3}(2 \varrho)-A_{4}(2 \varrho)\right\} \text {. }
$$

Diese Darstellung ist nun aber für die numerische Berechnung bei kleinem $\varrho$ schlecht geeignet. Wie man nämlich aus (2a) entnimmt, gehen die Funktionen $A_{n}(\varrho)$ für $\varrho \rightarrow 0$ wie $n ! / \varrho^{n+1}$ gegen $\infty$ und sind daher für kleine $\varrho$ sehr groß. $K_{2 \mathrm{~s} 2 \mathrm{~s}} / \zeta_{2 \mathrm{~s}}$ dagegen strebt für $\varrho \rightarrow 0$ dem endlichen Wert 0,5 zu. Man benötigt daher, wenn man für $K_{2 s 2 \mathrm{~s}}$ eine bestimmte Zahl von Dezimalstellen verlangt, eine mit abnehmendem $\varrho$ immer größer werdende Anzahl von geltenden Ziffern für die $A_{n}$. Das gleiche Verhalten ergibt sich auch dann, wenn man in (3a) für die $A_{n}$ ihre expliziten Darstellungen (2a) einsetzt. Man erhält so für das obige Beispiel

$$
\frac{K_{2 \mathrm{~s} 2 \mathrm{~s}}}{\zeta_{2 \mathrm{~s}}}=\frac{1}{\varrho}\left\{1-\left(1+\frac{3}{2} \varrho+\varrho^{2}+\frac{1}{3} \varrho^{3}\right) e^{-2 \varrho}\right\} .
$$

Da der zweite Term in der geschweiften Klammer für $\varrho \rightarrow 0$ gegen eins strebt, muß man denselben

${ }^{4}$ M. K ot an i u. Mitarb., J. Phys. Soc. Japan 8, 463 [1953]; 9, 553 [1954].

5 W. Bingel, Z. Naturforschg. 9 a, 675 [1954], Gl. (12). 
um so genauer berechnen, je kleiner $\varrho$ ist, um immer die gleiche Zahl von Dezimalstellen für $K_{2 \mathrm{~s} 2 \mathrm{~s}}$ zu bekommen.

Die folgende Auswertung von (1) vermeidet diesen Nachteil. Zunächst läßt sich nach $R$ o o th a a ${ }^{2}$ die Ladungsverteilung $a_{\alpha}(1) \cdot a_{\beta}(1)$ in eine Summe von Standardladungsverteilungen

$$
\begin{gathered}
{[N L M]=\left(\frac{2 L+1}{4 \pi}\right)^{1 / 2} \cdot \frac{2^{L}(2 \bar{\zeta})^{N+2}}{(N+L+1) !}} \\
\cdot r^{N-1} e^{-2 \zeta r} S_{L M}(\Theta, \varphi)
\end{gathered}
$$

entwickeln, wenn man für die $a_{\alpha}, a_{\beta} \mathrm{S} \mathrm{l}$ a te r $-\mathrm{AO}^{\prime}$ 's $^{5}$ verwendet. Dabei ist $\bar{\zeta}=\frac{1}{2}\left(\zeta_{\alpha}+\zeta_{\beta}\right)$ der mittlere „orbital exponent", $\zeta_{\alpha}=Z_{\alpha} / n_{\alpha}$ und $\zeta_{\beta}=Z_{\beta} / n_{\beta}$. $S_{L M}$ ist die auf Eins normierte reelle Kugelflächenfunktion

$$
\begin{aligned}
S_{L M}(\Theta, \varphi)= & {\left[\left(\frac{2 L+1}{2 \pi}\right) \cdot \frac{(L-|M|) !}{(L+|M|) !}\right]^{1 / 2} \quad(4 \mathrm{a}) } \\
& \cdot P_{L}^{|M|}(\cos \Theta) \cdot\left\{\begin{array}{l}
\cos |M| \varphi \\
\sin |M| \varphi
\end{array}, M \gtrless 0\right. \\
S_{L 0}(\Theta, \varphi)= & {\left[\frac{2 L+1}{4 \pi}\right]^{1 / 2} \cdot P_{L}(\cos \Theta) . }
\end{aligned}
$$

Das Integral (1) läßt sich dann durch die gleiche Summe von Standardintegralen

$$
\begin{aligned}
& {\left[\mathrm{b} \mid N L M_{\mathrm{a}}\right]=\int \frac{\left[N L M_{\mathrm{a}}\right]}{r_{\mathrm{b} 1}} \mathrm{~d} \tau_{1}} \\
& \begin{array}{r}
=\frac{2^{L}(2 \overline{\zeta)})^{N+2}}{(N+L+1) !} R^{N+1}\left\{\int_{0}^{1} \xi^{N+L+1} e^{-2}\right. \\
\text { as zweite Integral in }(7 \mathrm{~b}) \text { wird umgeformt } \\
\int_{1}^{\infty} \ldots=\int_{0}^{\infty} \ldots-\int_{0}^{1} \ldots=\frac{(N-L) !}{(2 \varrho)^{N-L+1}}-\int_{0}^{1} \cdots,
\end{array} \\
& {\left[\mathrm{b} \mid N L \Sigma_{\mathrm{a}}\right]=\frac{2^{L}(2 \bar{\zeta})^{N+2}}{(N+L+1) !} R^{N+1}\left\{\frac{(N-L) !}{(2 \varrho)^{N-L+1}}\right.} \\
& \left.+\int_{0}^{1}\left(\xi^{N+L+1}-\xi^{N-L}\right) e^{-2 \varrho \xi} \mathrm{d} \xi\right\} .
\end{aligned}
$$

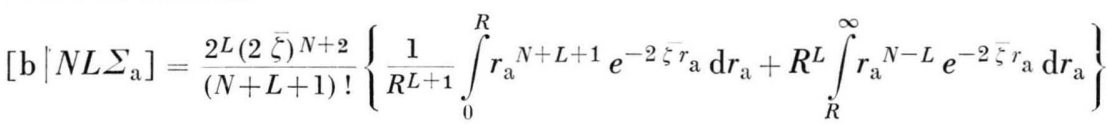

ausdrücken. Dieser Schritt wird auch bei der Auswertung in elliptischen Koordinaten zweckmäßigerweise zuerst vorgenommen ${ }^{2}$.

Wir führen dagegen Polarkoordinaten $r_{\mathrm{a}}, \Theta, \varphi$ mit a als Zentrum und ab als Polarachse ein. Durch die Entwicklung

$\left.\left.\frac{1}{r_{\mathrm{b} 1}}=\sum_{k=0}^{\infty} \frac{r_{<}^{k}}{r_{>}^{k+1}} P_{k}(\cos \Theta), \quad \begin{array}{l}r_{<} \\ r_{>}\end{array}\right\}=\begin{array}{l}\text { Minimum } \\ \text { Maximum }\end{array}\right\}\left(r_{\mathrm{a}}, R\right)$

in Verbindung mit (4), (4a) wird (5) zu

$$
\begin{aligned}
{\left[\mathrm{b} \mid N L M_{\mathrm{a}}\right]=\left(\frac{2 L+1}{4 \pi}\right)^{1 / 2} \cdot \frac{2^{L}(2 \bar{\xi})}{(N+L+2) !} } & \\
& \cdot \sum_{k=0}^{\infty} \int_{0}^{\infty} r_{\mathrm{a}}{ }^{N-1} \frac{r_{<}^{k}}{r_{>}^{k+1}} r_{\mathrm{a}}{ }^{2} \mathrm{~d} r_{\mathrm{a}} \\
& \cdot \int_{\text {Winkel }} P_{k}(\cos \Theta) \cdot S_{L M}(\Theta, \varphi) \mathrm{d} \Omega .
\end{aligned}
$$

Das zweite Integral über die Winkel ist nur für $M=0$ von Null verschieden ( $\varphi$-Integration) und hat dann den nur für $k=L$ von Null verschiedenen Wert $(4 \pi /(2 L+1))^{1 / 2}(\Theta$-Integration). Von der Summe über $k$ bleibt daher nur ein Term übrig (für $M=0$ steht $\Sigma$ )
Führt man jetzt die neue Hilfsfunktion

$$
\begin{aligned}
C_{n}(\varrho) & =\int_{0}^{1} \xi^{n} e^{-\varrho \xi} \mathrm{d} \xi=\frac{n !}{\varrho^{n+1}}-A_{n}(\varrho) \\
& =\sum_{k=0}^{\infty}(-1)^{k} \frac{\varrho^{k}}{k !(n+k+1)}
\end{aligned}
$$

ein, so wird endlich

$$
\begin{aligned}
& {\left[\mathrm{b} \mid N L \Sigma_{\mathrm{a}}\right] }=\bar{\zeta} \cdot \frac{2^{L+1}}{(N+L+1) !}\left\{(N-L) !(2 \varrho)^{L}\right. \\
&\left.+(2 \varrho)^{N+1}\left(C_{N+L+1}(2 \varrho)-C_{N-L}(2 \varrho)\right)\right\} .
\end{aligned}
$$

[b $\left.\mid N L M_{\mathrm{a}}\right]=0$ für $M \neq 0$.

Das ist die gesuchte Darstellung des Standardintegrals (5), ausgedrückt durch die Hilfsfunktionen $C_{n}(\varrho)$. Dieselben haben nach $(9 \mathrm{~b})$ für $\varrho=0$ den endlichen Wert $1 /(n+1)$, so daß die im Anschluß an Gl. (3a) geschilderte Schwierigkeit bei der numerischen Berechnung für kleine $\varrho$ hier nicht auftritt. Aus (10) erhält man für $\varrho=0$ die Standardform des Einzentrenintegrals ( 1 a)

$$
\left[\mathrm{a} \mid N L M_{\mathrm{a}}\right]=\bar{\zeta} \cdot \frac{2}{N+1} \delta_{0, L} \delta_{0, M} .
$$


Der zweite Term in (10) gibt zu (10a) wegen $N \geqq 1$ keinen, der erste nur für S-artige Ladungsverteilungen $(L=0)$ einen Betrag.

Gl. (10) ist wegen $(9 \mathrm{~b})$ eine Entwicklung nach positiven Potenzen des Kernabstandes $R(\varrho=\bar{\zeta} R)$, was für kleine $R$ natürlich die geeignetste Darstellung ist. Gl. (10) zeigt ferner, daß in der Entwicklung der ursprünglichen Ladungsverteilung $a_{\alpha}(1) \cdot a_{\beta}(1)$ nur die $\Sigma$-Terme $(M=0)$ zum Übergangsintegral (1) beitragen. Die folgenden Formeln geben alle von Null verschiedenen Übergangsintegrale $K_{\alpha \beta}$ zwischen allen AO's mit den Hauptquantenzahlen $n=1$ und 2. Dabei ist $K_{\alpha \beta}$ zuerst durch die Standardintegrale (5) und an zweiter Stelle mittels (10) durch die Hilfsfunktionen $C_{n}$ ausgedrückt. Für $L=0,1,2, \ldots$ stehen die üblichen aus der Theorie der Atomspektren bekannten Bezeichnungen $\mathrm{S}, \mathrm{P}, \mathrm{D} \ldots ;(p, q)$ ist eine Abkürzung für

$$
\left(\zeta_{\alpha} / \bar{\zeta}\right)^{p}\left(\zeta_{\beta} / \bar{\zeta}\right)^{q} \text { mit } \bar{\zeta}=\frac{1}{2}\left(\zeta_{\alpha}+\zeta_{\beta}\right),
$$

das nicht jedesmal angegebene Argument von $C_{n}$ ist $2 \varrho$.

$$
\begin{aligned}
& K_{1 \mathrm{~s} 1 \mathrm{~s}^{\prime}}=\left(\frac{3}{2}, \frac{3}{2}\right) \cdot\left[\mathrm{b} \mid 1 \mathrm{~S} \Sigma_{\mathrm{a}}\right]\left(\frac{3}{2}, \frac{3}{2}\right) \cdot \zeta \cdot\left[1-4 \varrho^{2}\left(C_{1}-C_{2}\right)\right], \\
& K_{1 \mathrm{~s} 2 \mathrm{~s}}=\left(\frac{3}{2}, \frac{5}{2}\right) \cdot \frac{\sqrt{3}}{2}\left[\mathrm{~b} \mid 2 \mathrm{~S} \Sigma_{\mathrm{a}}\right] \\
&=\left(\frac{3}{2}, \frac{5}{2}\right) \cdot \frac{\zeta}{\sqrt{3}} \cdot\left[1-4 \varrho^{3}\left(C_{2}-C_{3}\right)\right], \\
& K_{2 \mathrm{~s} 2 \mathrm{~s}^{\prime}}=\left(\frac{5}{2}, \frac{5}{2}\right) \cdot\left[\mathrm{b} \mid 3 \mathrm{~S} \Sigma_{\mathrm{a}}\right] \\
&=\left(\frac{5}{2}, \frac{5}{2}\right) \cdot \frac{\zeta}{2} \cdot\left[1-\frac{8}{3} \varrho^{4}\left(C_{3}-C_{4}\right)\right],
\end{aligned}
$$

$K_{1 \mathrm{~s} 2 \mathrm{p} \sigma}=\left(\frac{3}{2}, \frac{5}{2}\right) \cdot\left[\mathrm{b} \mid 2 \mathrm{P} \Sigma_{\mathrm{a}}\right]$

$$
=\left(\frac{3}{2}, \frac{5}{2}\right) \cdot \frac{\zeta}{3} \cdot\left[\varrho-4 \varrho^{3}\left(C_{1}-C_{4}\right)\right],
$$

$K_{2 \mathrm{~s}, 2 \mathrm{p} \sigma}=\left(\frac{5}{2}, \frac{5}{2}\right) \frac{5}{2 \downarrow 3}\left[\mathrm{~b} \mid 3 \mathrm{P} \Sigma_{\mathrm{a}}\right]$

$$
=\left(\frac{5}{2}, \frac{5}{2}\right) \cdot \frac{2 \zeta}{15} \cdot\left[\varrho-4 \varrho^{4}\left(C_{2}-C_{5}\right)\right],
$$

$\left.\begin{array}{c}K_{2 \mathrm{p} \sigma 2 \mathrm{p} \sigma^{\prime}} \\ K_{2 \mathrm{p} \pi 2 \mathrm{p} \pi^{\prime}}\end{array}\right\}=\left(\frac{5}{2}, \frac{5}{2}\right) \cdot\left(\left[\mathrm{b} \mid 3 \mathrm{~S} \Sigma_{\mathrm{a}}\right]+\left\{\begin{array}{c}3 \\ -8 / 2\end{array}\right\}\left[\mathrm{b} \mid 3 \mathrm{D} \Sigma_{\mathrm{a}}\right]\right)$ $=\left(\frac{5}{2}, \frac{5}{2}\right) \cdot \zeta \cdot\left\{\begin{array}{l}\frac{1}{2}+\frac{2}{15} \varrho^{2}\left(1+C_{1}-C_{6}\right)-\frac{4}{3} Q^{4}\left(C_{3}-C_{4}\right), \\ \frac{1}{2}-\frac{1}{15} \varrho^{2}\left(1+C_{1}-C_{6}\right)-\frac{4}{3} \varrho^{4}\left(C_{3}-C_{4}\right) .\end{array}\right.$

Die von $\varrho$ unabhängigen Terme in (11) geben gleichzeitig die Einzentren-Übergangsintegrale ( $1 \mathrm{a}$ ).

Tab. 1 gibt Zahlenwerte von $C_{n}(\varrho)$ für $n=0,1 \ldots 6$ und $\varrho=0(0,1) 1$. Sie wurden folgendermaßen berechnet. Aus (9a) läßt sich leicht die folgende Rekursionsformel für die $C_{n}(\varrho)$ ableiten

$$
\begin{aligned}
C_{n+1}(\varrho) & =\frac{1}{\varrho}\left\{(n+1) C_{n}(\varrho)-e^{-\varrho}\right\}, \text { speziell } \\
C_{0}(\varrho) & =\frac{1}{\varrho}\left\{1-e^{-\varrho}\right\} .
\end{aligned}
$$

\begin{tabular}{|c|c|c|c|c|c|c|c|}
\hline$\varrho$ & $C_{0}$ & $C_{1}$ & $C_{2}$ & $C_{3}$ & $C_{4}$ & $C_{5}$ & $C_{6}$ \\
\hline 0 & 1.000000 & 0.500000 & 0.333333 & 0.250000 & 0.200000 & 0.166667 & 0.142857 \\
\hline & 48374 & 32116 & 24027 & 19190 & 15973 & 13679 & 11961 \\
\hline 0,1 & 0.951626 & 467884 & 309306 & 230810 & 184027 & 152988 & 130896 \\
\hline & 45280 & 29807 & 22186 & 17659 & 14663 & 12534 & 10944 \\
\hline 0,2 & 906346 & 438077 & 287120 & 213151 & 169364 & 140454 & 119952 \\
\hline & 42407 & 27673 & 20491 & 16254 & 13462 & 11488 & 10016 \\
\hline 0,3 & 863939 & 410404 & 266629 & 196897 & 155902 & 128966 & 109936 \\
\hline & 39739 & 25704 & 18931 & 14963 & 12363 & 10528 & 9166 \\
\hline 0,4 & 824200 & 384700 & 247698 & 181934 & 143539 & 118438 & 100770 \\
\hline & 37261 & 23884 & 17495 & 13778 & 11354 & 9651 & 8391 \\
\hline 0,5 & 786939 & 360816 & 230203 & 168156 & 132185 & 108787 & 092379 \\
\hline & 34959 & 22201 & 16173 & 12690 & 10430 & 8849 & 7680 \\
\hline 0,6 & 751980 & 338615 & 214030 & 155466 & 121755 & 099938 & 084699 \\
\hline & 32816 & 20646 & 14954 & 11690 & 9582 & 8113 & 7032 \\
\hline 0,7 & 719164 & 317969 & 199076 & 143776 & 112173 & 091825 & 077667 \\
\hline & 30825 & 19207 & 13831 & 10770 & 8803 & 7423 & 6438 \\
\hline 0,8 & 688339 & 298762 & 185245 & 133006 & 103370 & 084402 & 071229 \\
\hline & 28972 & 17876 & 12798 & 9927 & 8095 & 6840 & 5896 \\
\hline 0,9 & 659367 & 280886 & 172447 & 123079 & 095275 & 077562 & 065333 \\
\hline 1,0 & $\begin{array}{r}27246 \\
632121\end{array}$ & $\begin{array}{r}16645 \\
264241\end{array}$ & $\begin{array}{r}11844 \\
160603\end{array}$ & $\begin{array}{r}9150 \\
113929\end{array}$ & $\begin{array}{r}7439 \\
087836\end{array}$ & $\begin{array}{r}6260 \\
071302\end{array}$ & $\begin{array}{r}5399 \\
059934\end{array}$ \\
\hline
\end{tabular}

Man könnte daher daran denken, zunächst $C_{0}$ nach (12 b) zu berechnen, um dann hieraus die höheren $C_{n}$ durch die Rekursionsformel (12 a) schrittweise zu bestimmen. Es zeigt sich jedoch, daß für das hier benötigte $\varrho$-Intervall der Abrundungsfehler sehr schnell mit $n$ anwächst, so daß man auf diese Weise die höheren $C_{n}$ mit geringerer Stellenzahl erhält als die niederen. Wir sind daher so vorgegangen, daß wir das höchste in der Tabelle vorkommende $C$,

Tab. 1. Hilfsfunktionen $C_{n}(\varrho)$. 
nämlich $C_{6}$, direkt aus seiner Potenzreihenentwicklung $(9 \mathrm{~b})$ berechnet haben, und dann die niederen $C_{n}$ durch Anwendung der Rekursionsformel (12 a) in umgekehrter Richtung, also in der Form

$$
C_{n-1}(\varrho)=\frac{1}{n}\left(\varrho C_{n}+e^{-\varrho}\right)
$$

bestimmten. Eine Probe bezüglich der Genauigkeit der so erhaltenen Werte ergab sich daraus, da $\beta$ die so berechneten $C_{0}$ bis auf wenige Einheiten der 7. Dezimale mit den nach $(12 \mathrm{~b})$ direkt berechneten übereinstimmten. Alle Zahlen wurden dann auf sechs Dezimalen abgerundet. Zur Erleichterung der Interpolation sind in der Tabelle auch die ersten Differenzen mit angegeben.

Als Beispiel der Überlegenheit der Darstellung (10) berechnen wir den Wert des Integrals $K_{2 \mathrm{~s} 2 \mathrm{~s}}$ für $\varrho=\frac{1}{8}$ und $\varrho=\frac{1}{4}$ mit den $A$-Funktionen ( 3 a), mit der expliziten Darstellung $(3 \mathrm{~b})$ und mit den $C$-Funktionen (10). In allen Fällen wird mit fünf geltenden Ziffern gerechnet. Man erhält dann

\begin{tabular}{|l|lll|}
\hline & \multicolumn{3}{|c|}{$K_{2 \mathrm{~s} 2 \mathrm{~s}} / \zeta_{2 \mathrm{~s}}$} \\
& nach (3 a) & nach (3 b) & nach (10) \\
\hline$\varrho=\frac{1}{8}$ & 0,49993 & 0,5000 & 0,49997628 \\
$\varrho=\frac{1}{4}$ & 0,4998 & 0,49980 & 0,4998126 \\
& & & $\left(\zeta_{\alpha}=\zeta_{\beta}=\zeta=\zeta_{2 \mathrm{~s}}\right)$ \\
\hline
\end{tabular}

Beide Male gibt (10) einen wesentlich genaueren Wert des Integrals. Oder, wollte man die gleiche Genauigkeit mit $(3 \mathrm{a}, \mathrm{b})$ erreichen, so müßte man dort die Ausgangswerte mit wesentlich mehr als fünf geltenden Ziffern in die Rechnung einführen.

Es kommt häufig vor, daß man die UUbergangs-Integrale für Werte der Variablen $\varrho$ benötigt, die nicht in den Tabellen enthalten sind.

Man muß also interpolieren. Nun zeigen aber (10), (11), daß in der Potenzreihenentwicklung nach $\varrho$ am Anfang eine Reihe von Potenzen fehlen. So verhält sich z. B. $K_{2 \mathrm{~s} 2 \mathrm{~s}}$ in der Umgebung von $\varrho=0$ wie

$$
K_{2 \mathrm{~s} 2 \mathrm{~s}}=C_{1}-C_{2} \varrho^{4}+\ldots,
$$

die Kurve $K_{2 \mathrm{~s} 2 \mathrm{~s}}(\varrho)$ verläuft also dort sehr flach. Das hat zur Folge, daß zwar die ersten Differenzen sehr klein sind, die zweiten und höheren Differenzen dagegen immer größer werden. Eine normale Interpolation ist hier gar nicht möglich, da diese voraussetzt, daß die höheren Differenzen sehr schnell kleiner werden, so daß man sie von einer gewissen Ordnung ab vernachlässigen kann. Die Darstellung (10) zeigt, wie man diese Schwierigkeiten vermeiden kann. Man interpoliert nicht die Standardintegrale selbst, sondern in den $C$-Funktionen, die sich gemäß (9 b) mit ihren Differenzen normal verhalten, was man auch aus der obigen Tabelle $\operatorname{der} C_{n}(\varrho)$ durch Bildung der zweiten Differenzen sieht. Bei fünf Dezimalen Genauigkeit wird man dabei im allgemeinen mit quadratischer Interpolation auskommen. Mit den so bestimmten $C_{n}$ berechnet man dann das gesuchte Integral nach (10) und (11).

\title{
Das Auflösungsvermögen des Feldionenmikroskopes
}

\author{
Von Erwin W. Mutller \\ Field Emission Laboratory, The Pennsylvania State University, University Park, U.S.A. \\ (Z. Naturforschg. 11 a, 88-94 [1956]; eingegangen am 17. November 1955)
}

\begin{abstract}
Eine Betrachtung des Auflösungsvermögens des Feldemissionsmikroskopes läßt den Betrieb mit Ionen an Stelle von Elektronen als vorteilhaft erscheinen. Einige Daten der Felddesorption und der Feldionenemission werden mitgeteilt und der Mechanismus dieser Effekte wird erläutert. Daraus ergibt sich eine Beziehung für das Auflösungsvermögen, die experimentell bestätigt wird. Helium erweist sich als besonders günstig für die Abbildung hochfester Metalloberflächen. Kühlung des Mikroskopes mit festem Stickstoff oder flüssigem Wasserstoff zur Erhöhung des Akkomodationskoeffizienten läßt die atomare Struktur des Objektes sichtbar werden. Benachbarte Wolframatome von 2,74 $\AA$ Abstand werden klar getrennt.
\end{abstract}

\section{Das Auflösungsvermögen des Feldelektronen-} mikroskopes

Der kleinste Streukreisdurchmesser oder das Auflösungsvermögen ${ }^{1,2}$ eines Feldelektronenmikroskopes (FEM) ist durch die Tangentialgeschwindig- keit und die Beugung der Elektronen begrenzt. Die Wirkung der Beugung kann auch durch die Heisen-

1 E. W. M ülle r, Z. Phys. 120, 270 [1943].

2 E. W. M üll e r, Ergebn. Exak. Naturw. XXVII, 290 [1953]. Siehe auch R. H. G o o d, jr. u. E. W. M üll er, Handbuch der Physik, 2. Auflage, Band 21, 1956. 